\title{
Annealing of radiation induced defects in silicon in a simplified phenomenological model
}

\author{
S. Lazanu ${ }^{\mathrm{a}}$ and I. Lazanu ${ }^{\mathrm{b}}$ \\ ${ }^{a}$ National Institute for Materials Physics, P.O.Box MG-7, Bucharest-Magurele, \\ Romania, electronic address: lazanu@alpha1.infim.ro \\ ${ }^{\mathrm{b}}$ University of Bucharest, Faculty of Physics, P.O.Box MG-11, \\ Bucharest-Magurele, Romania, electronic address: ilaz@scut.fizica.unibuc.ro
}

\begin{abstract}
The concentration of primary radiation induced defects has been previously estimated considering both the explicit mechanisms of the primary interaction between the incoming particle and the nuclei of the semiconductor lattice, and the recoil energy partition between ionisation and displacements, in the frame of the Lindhard theory. The primary displacement defects are vacancies and interstitials, that are essentially unstable in silicon. They interact via migration, recombination, annihilation or produce other defects. In the present work, the time evolution of the concentration of defects induced by pions in medium and high resistivity silicon for detectors is modelled, after irradiation. In some approximations, the differential equations representing the time evolution processes could be decoupled. The theoretical equations so obtained are solved analytically in some particular cases, with one free parameter, for a wide range of particle fluences and/or for a wide energy range of the incident particles, for different temperatures; the corresponding stationary solutions are also presented.
\end{abstract}

PACS:

61.80.Az: Theory and models of radiation effects

61.70.At: Defects formation and annealing processes

Key words: radiation damage, pions, atom displacements, kinetics of defects, annealing processes

\section{Introduction}

A point defect in a crystal is an entity that causes an interruption in the lattice periodicity. In this paper, the terminology and definitions in agreement with 
M. Lannoo and J. Bourgoin [1] are used in relation to defects.

Vacancies and interstitials are produced in materials exposed to radiation in equal quantities; they are the primary radiation defects, being produced either by the incoming particle, or as a consequence of the subsequent collisions produced by the primary recoil

In previous papers $[2,3]$ we calculated the concentration of radiation induced primary defects (CPD) in semiconductors exposed to hadron irradiation. Due to the important weight of annealing processes, as well as to their very short time scale, CPD is not a measurable physical quantity.

In silicon, vacancies and interstitials are essentially unstable and interact via migration, recombination, annihilation or produce other defects. The system evolves toward natural equilibrium.

The problem of the annealing of radiation induced defects in semiconductor materials is not new. Several models, empirical or more theoretic, have been previously proposed to explain these phenomena. Damask and Dienes developed, in the 1960s, a theoretical model that considers, as distinct cases, the kinetics of vacancy-interstitial annihilation with interstitial trapping at impurities [4], of vacancy-interstitial annihilation with interstitial trapping at impurities [5], and of vacancy-interstitial annihilation with diinterstitial formation [6]. In the first two cases, analytical solutions have been obtained by the authors, while in the last case only numerical solutions are possible. The escape of interstitials and vacancies from clusters and their subsequent interactions have been modelled by Tsveybak et al. [7]. Phenomenological approaches of the annealing could be found in Moll et al. [8] and Bates and co-workers [9] and references cited therein.

Most of the old studies were dedicated to electronic devices, made from low resistivity (in the $\Omega \mathrm{cm}$ range) silicon. Recently, the behaviour of the silicon material with medium and high resistivity (tens $\Omega c m-k \Omega c m$ ) in intense hadron fields started to present very much interest, these materials representing a major option for the detectors at the new generation of particle colliders and for space applications.

In the present paper, the time evolution of the concentration of primary defects induced by pion irradiation in silicon is studied in the frame of a simplified phenomenological model based on direct interactions between the primary induced defects and the impurities present in the material. 


\section{Equations for the kinetics of radiation induced defects: general formulation}

The starting hypothesis of the physical model is that the irradiation process is unique at the determined particle fluence, and that the time of irradiation is shorter that all characteristic times of annealing.

In the frame of the model, equal concentrations of vacancies and interstitials are supposed to be produced by irradiation, in much greater concentrations than the corresponding thermal equilibrium values, characteristic to each temperature. Both the pre-existing defects and those produced by irradiation, as well as the impurities, are assumed to be randomly distributed in the solid. An important part of the vacancies and interstitials annihilate. The sample contains certain concentrations of impurities which can trap interstitials and vacancies respectively, and form stable defects.

In the present paper, vacancy-interstitial annihilation, interstitial migration to sinks, vacancy and interstitial impurity complex formation as well as divacancy formation are considered. The sample could contain more impurities that trap vacancies or interstitials, and in this case all processes are to be taken into account.

The following notations are used: $V$ - monovacancy concentration; $I$ - free interstitial concentration, $J_{1}$ - total impurity "1" concentration (impurity "1" traps interstitials and forms the complex $C_{1}$ ); $C_{1}$ - interstitial-impurity concentration: one interstitial trapped for one complex formed; $J_{2}$ - total impurity "2" concentration (impurity "2" traps vacancies and forms the complex $C_{2}$ ); $C_{2}$ - vacancy-impurity concentration: one vacancy trapped for one complex formed; $V_{2}$ - divacancy concentration. All concentrations are expressed as atomic fractions.

The chemical scheme for reaction rates has been used, and the differential equations corresponding to the above picture are:

$$
\begin{gathered}
\frac{d V}{d t}=-K_{1} V I-K_{5} V\left(J_{20}-C_{2}\right)+K_{6} C_{2}-K_{7} V^{2}+K_{8} V_{2} \\
\frac{d I}{d t}=-K_{1} V I-K_{2} I-K_{3} I\left(J_{10}-C_{1}\right)+K_{4} C_{1}
\end{gathered}
$$




$$
\begin{gathered}
\frac{d C_{1}}{d t}=K_{3} I\left(J_{10}-C_{1}\right)-K_{4} C_{1} \\
\frac{d C_{2}}{d t}=K_{5} V\left(J_{20}-C_{2}\right)-K_{6} C_{2} \\
\frac{d V_{2}}{d t}=\frac{1}{2} K_{7} V^{2}-\frac{1}{2} K_{8} V_{2}
\end{gathered}
$$

The bimolecular recombination law of interstitials and vacancies is supposed to be a valid approximation for the present discussion, because at the concentrations of vacancies of interest, only a small fraction of defects anneal by correlated annihilation if their distribution is random (see the discussion in $[10])$.

If $N$ is the total defect concentration, expressed as atomic fraction:

$$
N=V+I+2 V_{2}+C_{1}+C_{2}
$$

then it satisfies the differential equation:

$$
\frac{d N}{d t}=-2 K_{1} V I
$$

The initial conditions, at $t=0$, are: at the end of the irradiation, there are equal concentrations of interstitials and vacancies: $I_{0}=V_{0}$; the concentrations of impurities are $J_{10}$ and $J_{20}$ respectively, there are no complexes in the sample: $C_{10}=C_{20}=0$, and no divacancies $V_{20}=0$.

The reaction constants $K_{1}$ and $K_{3}$ are determined by the diffusion coefficient for the interstitial atom to a substitutional trap, and therefore $K_{1}=K_{3}$ :

$$
K_{1}=30 \nu \exp \left(-E_{i 1} / k_{B} T\right)
$$


where $E_{i 1}$ is the activation energy of interstitial migration and $\nu$ the vibrational frequency. The reaction constant $K_{2}$ (related to interstitial migration to sinks) is proportional to the sink concentration $\alpha$ :

$$
K_{2}=\alpha \nu \lambda^{2} \exp \left(-E_{i 1} / k_{B} T\right)
$$

with $\lambda$ the jump distance.

$K_{4}$ characterises the decomposition of a complex into an impurity and an interstitial, and is given by:

$$
K_{4}=5 \nu \exp \left(\frac{E_{i 1}+B_{1}}{k_{B} T}\right)
$$

$K_{5}$ and $K_{6}$ describe the formation and decomposition of vacancy-impurity complexes, while $K_{7}$ and $K_{8}$ the formation and decomposition of divacancies. and are given by:

$$
K_{5}=30 \nu \exp \left(-E_{i 2} / k_{B} T\right)
$$

with $E_{i 2}$ the activation energy for vacancy migration;

$$
\begin{aligned}
& K_{6}=5 \nu \exp \left(-\frac{E_{i 2}+B_{2}}{k_{B} T}\right) \\
& K_{7}=30 \nu \exp \left(-E_{i 2} / k_{B} T\right) \\
& K_{8}=5 \nu \exp \left(-\frac{E_{i 2}+B_{3}}{k_{B} T}\right)
\end{aligned}
$$

where $B_{1}$ is the binding energy of $C_{1}, B_{2}$ the binding energy of $C_{2}$ and $B_{3}$ is the corresponding binding energy of divacancies. 
Due to the mathematical difficulties to solve analytically the complete differential equation system, some simplifications are necessary.

\section{$3 \quad$ Hypothesis, approximations and discussions}

The interstitials are much more mobile in silicon in respect to vacancies, and are characterised by an activation energy of migration a factor of two times smaller.

This difference between the activation energies $E_{i 1}$ and $E_{i 2}$ respectively justifies the introduction of two time scales, and the separate study of the processes involving interstitials and vacancies respectively. In the first stage, vacancy interstitial annihilation and interstitials migration to sinks are studied. The concentration of interstitials decays much rapidly than the concentration of vacancies. A cut-off condition for $I$ is imposed (a $p$ times decrease of interstitial concentration, $p$ being an adjustable parameter of the model). The vacancy concentration determined by this procedure is the initial value for the processes comprised in the second stage, and will be denoted by $V_{\text {initial }}$. The formation of complexes by vacancies is considered less important, and is neglected in the following discussion.

So, for the "first stage", after some simple manipulations, from (1) and (2), the equation:

$$
\frac{d I}{d t}=1+\frac{K_{2}}{K_{1} V}
$$

has been obtained, with the solution:

$$
I=V+\frac{K_{2}}{K_{1}} \ln \frac{V}{V_{0}}
$$

and:

$$
t=\frac{\ln \left[1+\frac{K_{1} V(t)}{K_{2} \ln (V(t))}\right]}{K_{2} \ln (V(t))}
$$


Imposing the cut-off condition for the concentration of interstitials, both $V_{\text {initial }}$ and the characteristic time could be found. As specified, $V_{\text {initial }}$ is used as initial vacancy concentration for the second step in the analysis, where the equations (4) and (5) are considered. $V_{\text {initial }}$ depends on $V_{0}$ and $p$, and it is temperature independent. This system of equations, expressing the kinetics of divacancy and vacancy-impurity formation, have no analytical solution. These processes are governed by the initial concentrations of vacancies and impurities.

If the impurity that traps vacancies is phosphorus, the limiting cases correspond to low initial doping concentration (high resistivity, uncompensated materials) and very high impurity concentration (low resistivity), respectively. In both cases the equations could be decoupled, and analytical solutions are possible.

So, if the formation of vacancy-impurity complexes is not so important, the main process responsible for the decay of vacancy concentration is divacancy production. In this case, the time evolution of the vacancy concentration is described by the equation:

$$
V(t)=\frac{1}{4 K_{7}}\left\{-K_{8}+R \frac{\frac{K_{8}+4 K_{7} V_{i}}{R}+\tanh \left(\frac{t R}{4}\right)}{1+\frac{K_{8}+4 K_{7} V_{i}}{R} \tanh \left(\frac{t R}{4}\right)}\right\}
$$

where:

$$
R \equiv \sqrt{K_{8}\left(K_{8}+8 K_{7} V_{i}\right)}
$$

while the increase of the divacancy concentration is given by:

$$
V_{2}(t)=\frac{V_{i}-V(t)}{2}
$$

The stationary solution for $V(t)$ is given by:

$$
\lim _{t \rightarrow \infty} V(t)=\frac{1}{4 K_{7}}\left(R-K_{8}\right)
$$

For n-type high doped Si, the process described by eq. (4) is the most probable. 
If $J_{0}$ is the initial concentration of impurities, and the initial concentration of complexes is zero, than:

$$
V(t)=\frac{1}{2 K_{5}}\left\{-K_{6}+K_{5}\left(V_{i}-J_{0}\right)+R^{*} \frac{\frac{K_{6}+K_{5}\left(V_{i}-J_{0}\right)}{R^{*}}+\tanh \left(\frac{t R^{*}}{2}\right)}{1+\frac{K_{6}+K_{5}\left(V_{i}-J_{0}\right)}{R^{*}} \tanh \left(\frac{t R^{*}}{2}\right)}\right\}
$$

with:

$$
R^{*} \equiv \sqrt{K_{6}^{2}+K_{5}^{2}\left(V_{i}-J_{0}\right)^{2}+2 K_{5} K_{6}\left(V_{i}+J_{0}\right)}
$$

and with the stationary solution:

$$
\lim _{t \rightarrow \infty} V(t)=\frac{1}{2 K_{5}}\left[K_{5}\left(V_{i}-J_{0}\right)+R^{*}-K_{6}\right]
$$

and

$$
C=V_{i}-V
$$

\section{Results and physical interpretations}

In Figure 1, the absolute value of the CPD per unit fluence (CPD) induced by pions in silicon is presented as a function of the kinetic energy of the particle. This curve has been obtained in the hypothesis listed in references $[11,12]$. In the kinetic energy range between 100 to $300 \mathrm{MeV}$, pion - nucleus interaction is resonant in all waves, the $\Delta_{33}$ resonance is produced and a pronounced maxima in the CPD is observed at approximately $150 \mathrm{MeV}$ followed by a slight monotonic decrease at higher energies. Some local maxima are also presents at high energies, but with less importance.

The process of partitioning the energy of the recoil nuclei (produced due the interaction of the incident particle with the nucleus, placed in its lattice site) in new interaction processes, between electrons (ionisation) and atomic motion (displacements) has been considered in the frame of the Lindhard theory. 
The CPD multiplied by the fluence is the initial value of the concentration of vacancies and interstitials, and in the forthcoming discussion it is expressed, as specified, as atomic fraction. Without loss of generality, will shall consider that the primary defects are produced by $150 \mathrm{MeV}$ kinetic energy pions.

The following values of the parameters have been used: $E_{i 1}=0.4 \mathrm{eV}, E_{i 2}=$ $0.8 \mathrm{eV}, B_{1}=0.2 \mathrm{eV}, B_{2}=0.2 \mathrm{eV}, B_{3}=0.4 \mathrm{eV}, \nu=10^{13} \mathrm{~Hz}, \lambda^{2}=10^{15} \mathrm{~cm}^{2}$, $\alpha=10^{10} \mathrm{~cm}^{-2}$, The value of the parameter $p$ is taken 100 .

The time evolution of the concentration of vacancies, normalised to the concentration of vacancies created by irradiation is represented in Figure 2. Fluences in the range $10^{11}-10^{15}$ pions $/ \mathrm{cm}^{2}$ have been considered. The weight of the annihilation process in respect to interstitial migration to sinks increases abruptly with the fluence. At low fluences, up to $10^{11}$ pions $/ \mathrm{cm}^{2}$, the annihilation has a low importance. With a good approximation, it could be considered that after $0.2 \mathrm{sec}$. after irradiation the vacancy concentration is saturated. As could be seen from the figure, observable effects in $V / V_{0}$ appear for fluences higher than $10^{12}$ pions $/ \mathrm{cm}^{2}$. Experimentally, for silicon particle detectors irradiated with hadrons, some modifications in the electrical characteristics have been observed in the fluence range $10^{12}-10^{13} \mathrm{~cm}^{-2}[13,14]$.

All the curves correspond to room temperature $\left(20{ }^{\circ} \mathrm{C}\right)$ annealing.

The interval of time after which the concentration of interstitials decreases $p$ times, given by eq. (17) with $V(t)=V_{\text {initial }}$ is a characteristic time of the processes in the first stage, and depends on temperature and fluence.

In Figure 3, this characteristic time is represented as a function of fluence, in the fluence range $10^{11}-10^{15}$ pions $/ \mathrm{cm}^{2}$, for three temperatures of interest for high energy physics applications: $-20,0$ and $+20{ }^{\circ} \mathrm{C}$. It may be seen that, up to $10^{13}$ pions $/ \mathrm{cm}^{2}$, the characteristic time is independent on the fluence, and for higher fluences a decrease of the characteristic time is to be noted and a fluence dependence is obtained.

The initial concentration of vacancies for the second stage is $V_{\text {initial }}$ previously calculated. The second stage starts at a time equal to the characteristic time.

Two limiting cases have been studied, low doping concentration (high resistivity uncompensated material), and low resistivity, phosphorus doped silicon. The formation and decomposition of divacancies is a good approximation for the first, while the second is related to $V-P$ complexes.

The time evolution of the concentration of vacancies when divacancy formation is considered is illustrated in Figure 4a. As underlined in Section 3, the time scale of this process is much longer in respect to the first stage. The origin of time in Figure $4 \mathrm{a}$ is the beginning of the second stage, this means a 
characteristic time after the start of the annealing.

The curves in this figure are normalised to the concentration of vacancies remained after the first stage. Values of the initial concentrations of vacancies that correspond to fluences in the same range as in the previous analysis have been considered. It could be observed that the time after which $V(t) / V_{\text {initial }}$ saturates is in the range $10^{7}-10^{8}$ sec., and increases with the irradiation fluence. All curves have been calculated also for $20^{\circ} \mathrm{C}$ temperature.

The time evolution of divacancy concentration is represented in Figure 4b, also in a similar manner with the vacancy concentration in Figure 4a.

The case of high initial impurity (phosphorus) concentration, where divacancy formation is neglected in respect to complex formation, is presented in Figures $5 \mathrm{a}$ and $5 \mathrm{~b}$ respectively.

Three initial concentrations for phosphorus in silicon (expressed as atomic fractions) have been considered: $10^{-5}, 10^{-7}$, and $5 \cdot 10^{-10}$, corresponding to 0.1 $\Omega \mathrm{cm}, 10 \Omega \mathrm{cm}$, and $1 \mathrm{k} \Omega \mathrm{cm}$ resistivity respectively. The time evolution of the concentration of vacancies is represented in Figure 5a, while the corresponding one for the concentration of complexes in Figure 5b, both normalised to the concentration of vacancies remained after the first stage. An irradiation with $10^{13}$ pions $/ \mathrm{cm}^{2}$ has been considered.

The origin of time in Figures $5 \mathrm{a}$ and $5 \mathrm{~b}$ is displaced in respect with the origin of the annealing, in the same way as in Figs. $4 \mathrm{a}$ and $4 \mathrm{~b}$.

The curves corresponding to $10^{11}-10^{15} \mathrm{~cm}^{-2}$ are superimposed on the same curve, with the exception of the tail at times longer than $10^{4} \mathrm{sec}$. where the values corresponding to higher fluences are slightly higher.

The time interval after which $V(t) / V_{\text {initial }}$ saturates is shorter than in the case of divacancy, and at room temperature is in the range $10^{3}-10^{4} \mathrm{sec}$. for the doping concentrations considered. It shortens with the increase of impurity concentration.

Some explicit considerations must be done about the formation of vacancyimpurity complexes. The mechanisms supposed above can be used both for boron and for phosphorus impurities. In this case, the corresponding processes are [8]:

$$
B_{i}+V \rightarrow\left[B_{i}-V\right]
$$

and respectively: 


$$
P_{s}+V \rightarrow\left[P_{s}-V\right]
$$

While the complex formed by boron is unstable and self anneals bellow room temperature, the interaction between a $V$ and a $P_{s}$ leads to the formation of an $E$ centre which is stable in the same conditions [7].

Interactions between interstitial oxygen (another very studied impurity in the last time) and free vacancies is described as a higher order process (third order in the [8] and fourth power in [15]). If the process is stopped as a first order one, the time evolution of the concentrations is not different from the case of phosphorus, studied before.

If two or more impurities that trap vacancies are considered as existing simultaneously in silicon, the system of coupled equations must be solved. Only numerical solutions for particular cases are possible.

If two or more impurities that trap vacancies are considered as existing simultaneously in silicon, the system of coupled equations must be solved. Only numerical solutions for particular cases are possible.

So, in the frame of this model, three characteristic times have been found for $20^{\circ} \mathrm{C}$ self annealing in silicon: one in the order of $0.2 \mathrm{sec}$, corresponding to vacancy - interstitial annihilation and interstitial migration to sinks, one around $10^{4}$ sec., related to vacancy-phosphorous formation and decomposition, and another one around $10^{7}$ sec., divacancy related.

The present model could be applied to the annealing of primary radiation defects induced in Si by any other hadron, the difference coming from the value of the CPD.

\section{Summary}

The time evolution of the primary concentration of defects induced by pions after irradiation process has been modelled.

In this model, vacancy-interstitial annihilation, interstitial migration to sinks, vacancy and interstitial impurity complex formation as well as divacancy formation have been considered. In the considered hypothesis, the time evolution of impurity concentrations has been decoupled into two steps, the first one involving vacancy-interstitial annihilation and interstitial migration to sinks, the second vacancy-complex and divacancy formation. 
The equations corresponding to the first step have been solved analytically for a wide range of particle fluences and for a wide energy range of incident particles, and for three different temperatures: $-20,0$ and $+20{ }^{\circ} \mathrm{C}$.

The approximations that permit to decouple the differential equations representing the time evolution processes in the second step have been studied, and the processes have been treated separately. The simultaneous consideration of more processes in this last step of the model is possible only numerically.

The predictions of the model are in general agreement with the experimental results, and suggest a possible connection with the self annealing mechanisms.

\section{Acknowledgements}

The authors are very grateful to Professor Gh. Ciobanu from the Bucharest University for helpful discussions during the course of this work.

\section{References}

[1] M. Lannoo, J. Bourgoin, "Point Defects in Semiconductors", Springer Series in Solid State Science 2, Eds. M. Cardona, P. Fulde, H.-J. Queisser, SpringerVerlag 1981.

[2] I. Lazanu, S. Lazanu, Nucl. Instr. and Meth. in Phys. Res. A 432 (1999) 374.

[3] I. Lazanu, S. Lazanu, E. Borchi, M. Bruzzi, Nucl. Instr. and Meth. in Phys. Res. A 406 (1998) 259.

[4] A. C. Damask, G. J. Dienes, Phys. Rev. 125 (1962) 444.

[5] G. J. Dienes, A. C. Damask, Phys. Rev. 125 (1962) 447.

[6] G. J. Dienes, A. C. Damask Phys. Rev. 128 (1962) 2542.

[7] I. Tsveybak, W. Bugg, J. A. Harvey, J. Walker, IEEE Trans. Nucl. Sci. NS-39 (1992) 1720.

[8] M. Moll, H. Feick, E. Fretwurst, G. Lindstrom, T. Schultz, Nucl. Phys. (Proc. Suppl.) 44B (1998) 468.

[9] S. J. Bates, C. Furetta, M. Glaser, F. Lemeilleur, C. Soave, E. Leon-Florian, Nucl. Phys. (Proc. Suppl.) 44B (1998) 510.

[10] A. C. Damask, G. J. Dienes, "Point Defects in Metals", Gordon and Breach, New York 1963. 
[11] S. Lazanu, I. Lazanu, Nucl. Instr. and Meth. in Phys. Res. A 419 (1998) 570.

[12] S. Lazanu, I. Lazanu, U. Biggeri, E. Borchi, M. Bruzzi, Nucl. Phys. (Proc. Suppl.) 61B (1998) 409.

[13] C. Leroy, P. Roy, G. Casse, M. Glaser, E. Grigoriev, F. Lemeilleur, Nucl. Instr. and Meth. in Phys. Res. A 426 (1999) 99.

[14] B. Dezillie, V. Eremin, Z. Li, Nucl. Instr. and Meth. in Phys. Res. A 426 (1999) 114.

[15] H. Reiss, Journal of App. Phys. 30 (1959) 141.

[16] Z. Li, W. Chen, H. W. Kraner, Nucl. Instr. and Meth. in Phys. Res. A 308 (1991) 585.

[17] F. Anghinolfi et al., RD2 Collaboration, Nucl. Instr. and Meth. in Phys. Res. A 326 (1993) 365. 


\section{Figure captions}

Figure 1: Energy dependence of the absolute value of the concentration of primary defects per unit fluence induced by pions in silicon.

Figure 2: Concentration of vacancies created by irradiation versus time, normalised to the concentration of vacancies for the first stage, for different particle fluences, expressed in $\mathrm{cm}-2$.

Figure 3: Characteristic time of the first stage versus the irradiation fluence, for $-20,0$ and $+20^{\circ} \mathrm{C}$ temperature.

Figure 4: a) Time evolution of the concentration of vacancies, normalised to the concentration of vacancies remained after the first stage when divacancy formation and decomposition are considered, for different particle fluences, expressed in $\mathrm{cm}^{-2}$. b) Same as (a) for the concentration of divacancies.

Figure 5: a) Time evolution of the concentration of vacancies, normalised to the concentration of vacancies remained after the first stage when $V-P$ complex formation and decomposition are considered. The three curves correspond to different initial impurity concentrations, expressed in atomic fractions. b) Same as (a) for the concentration of complexes. 


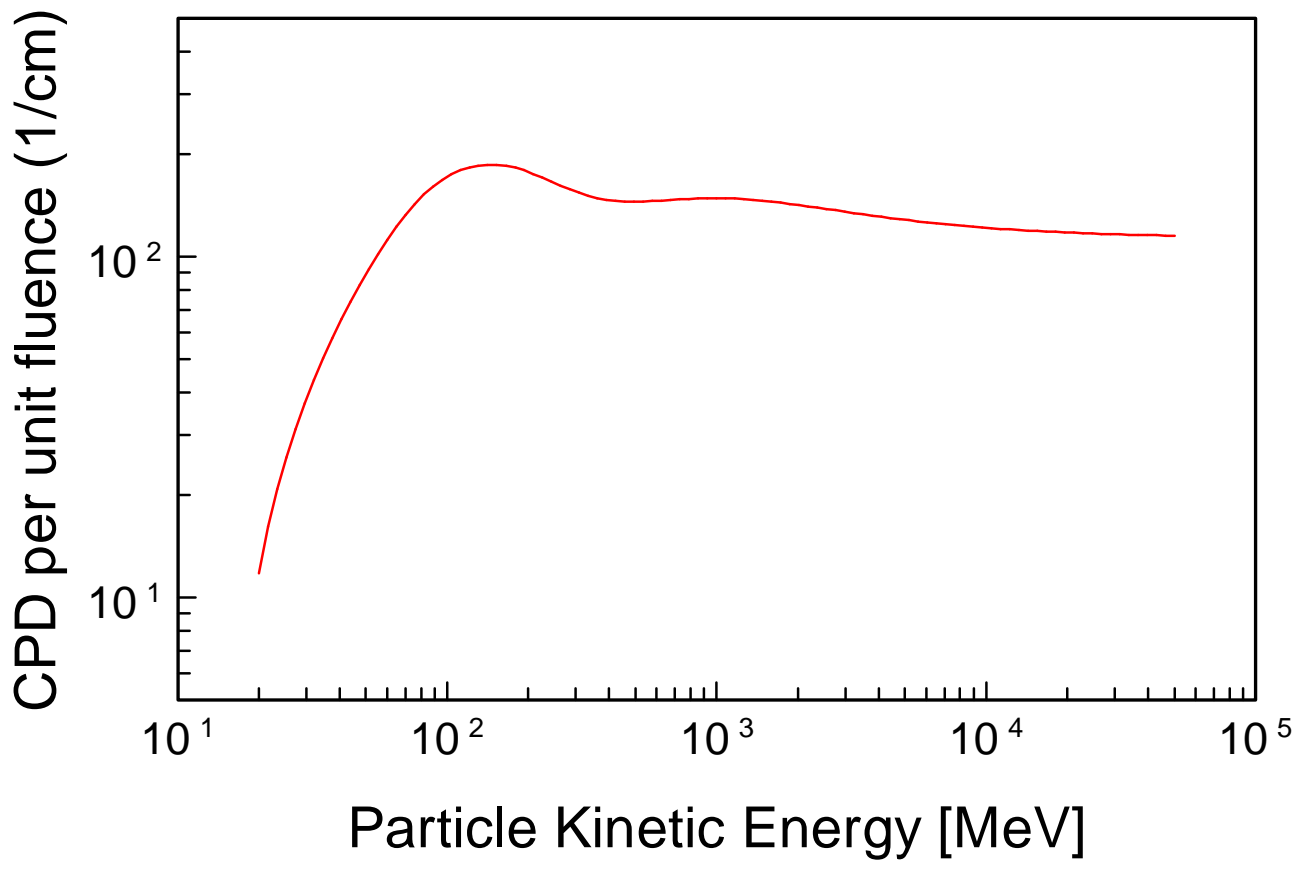




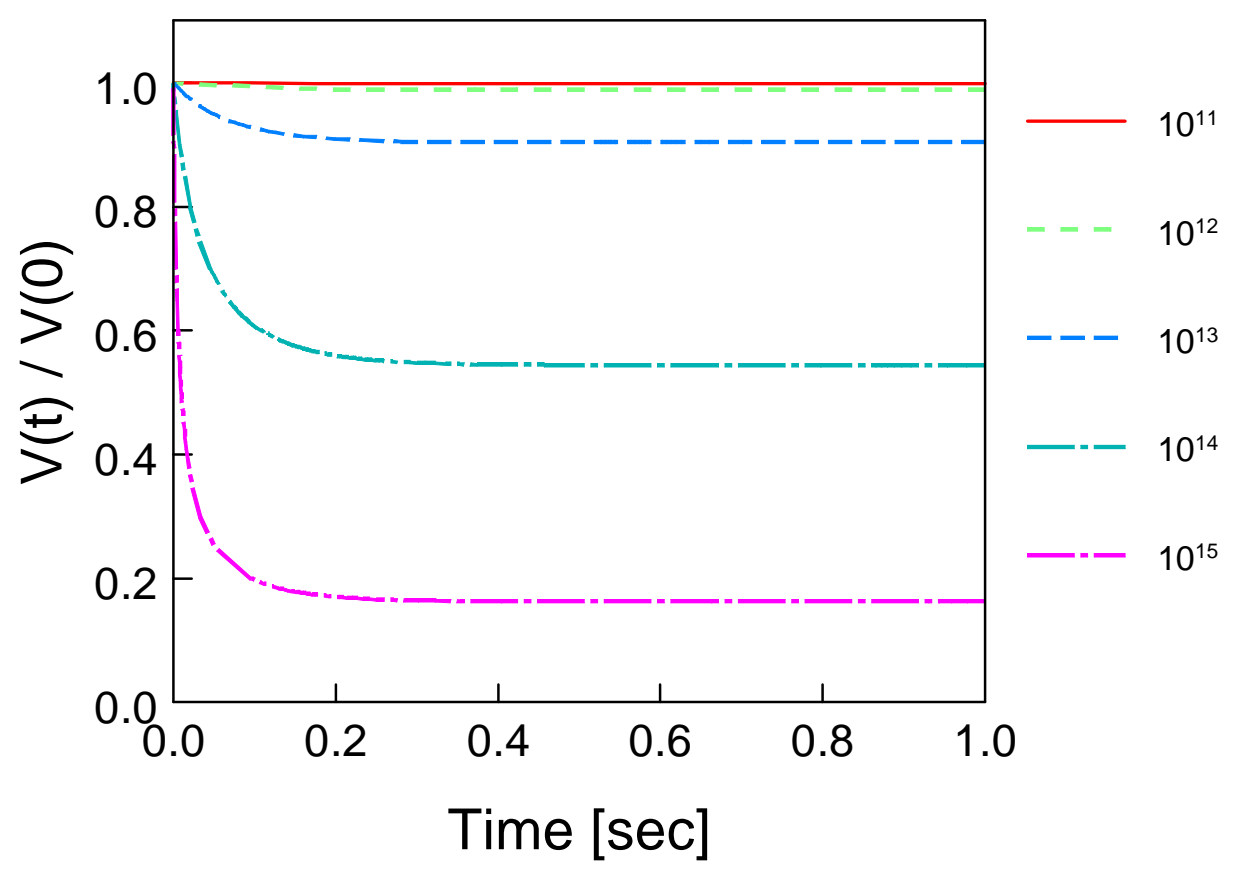




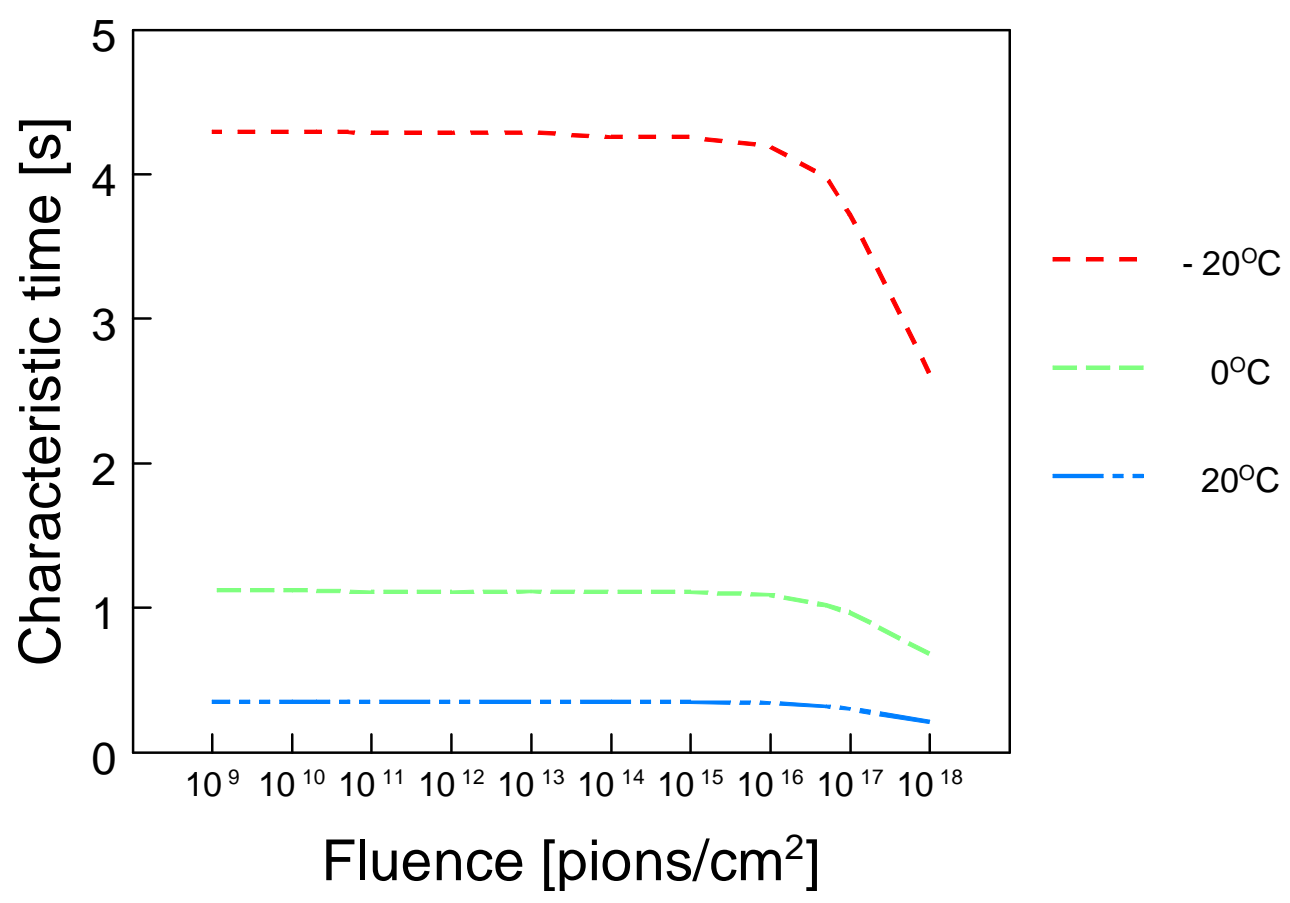



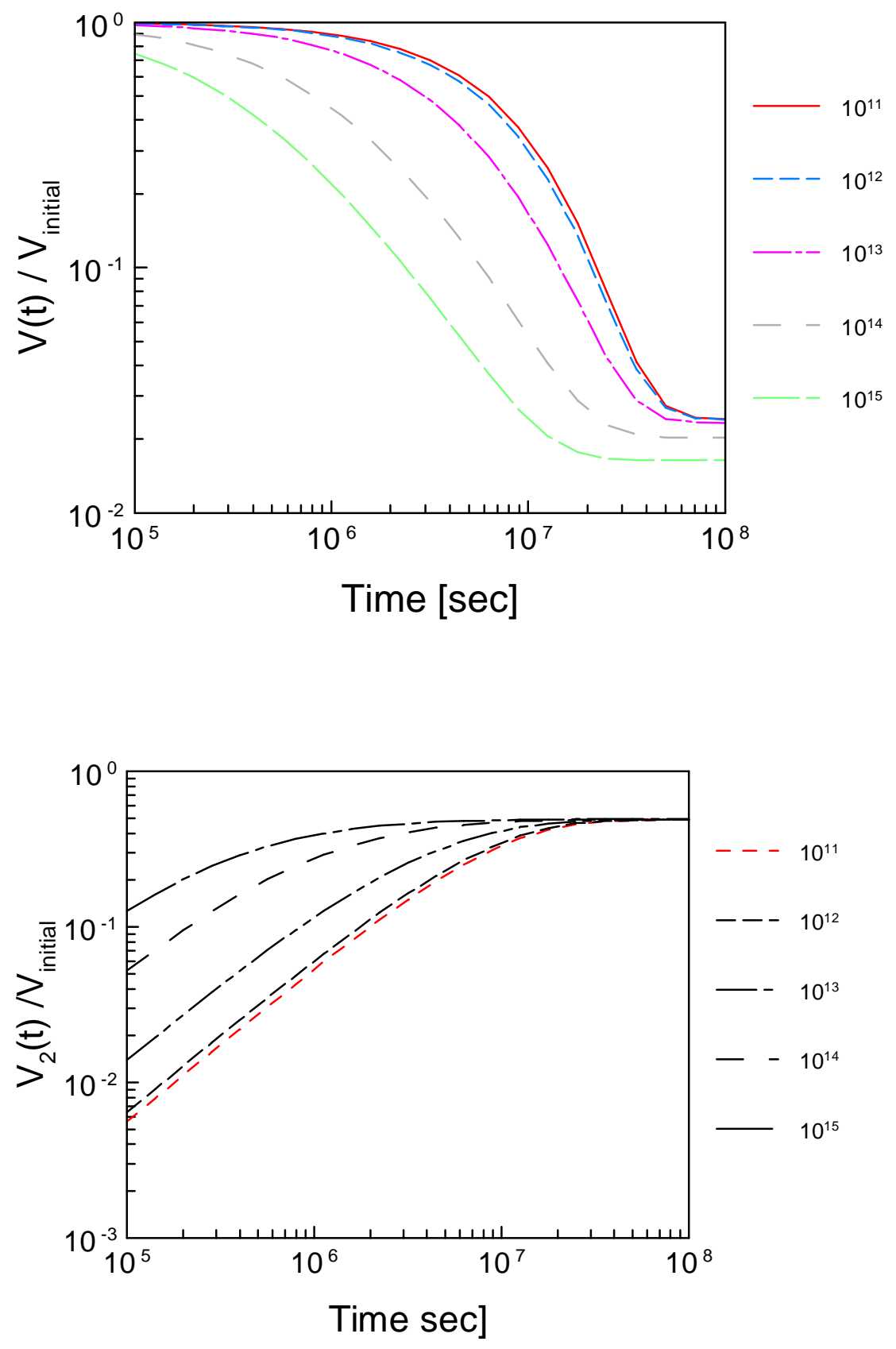

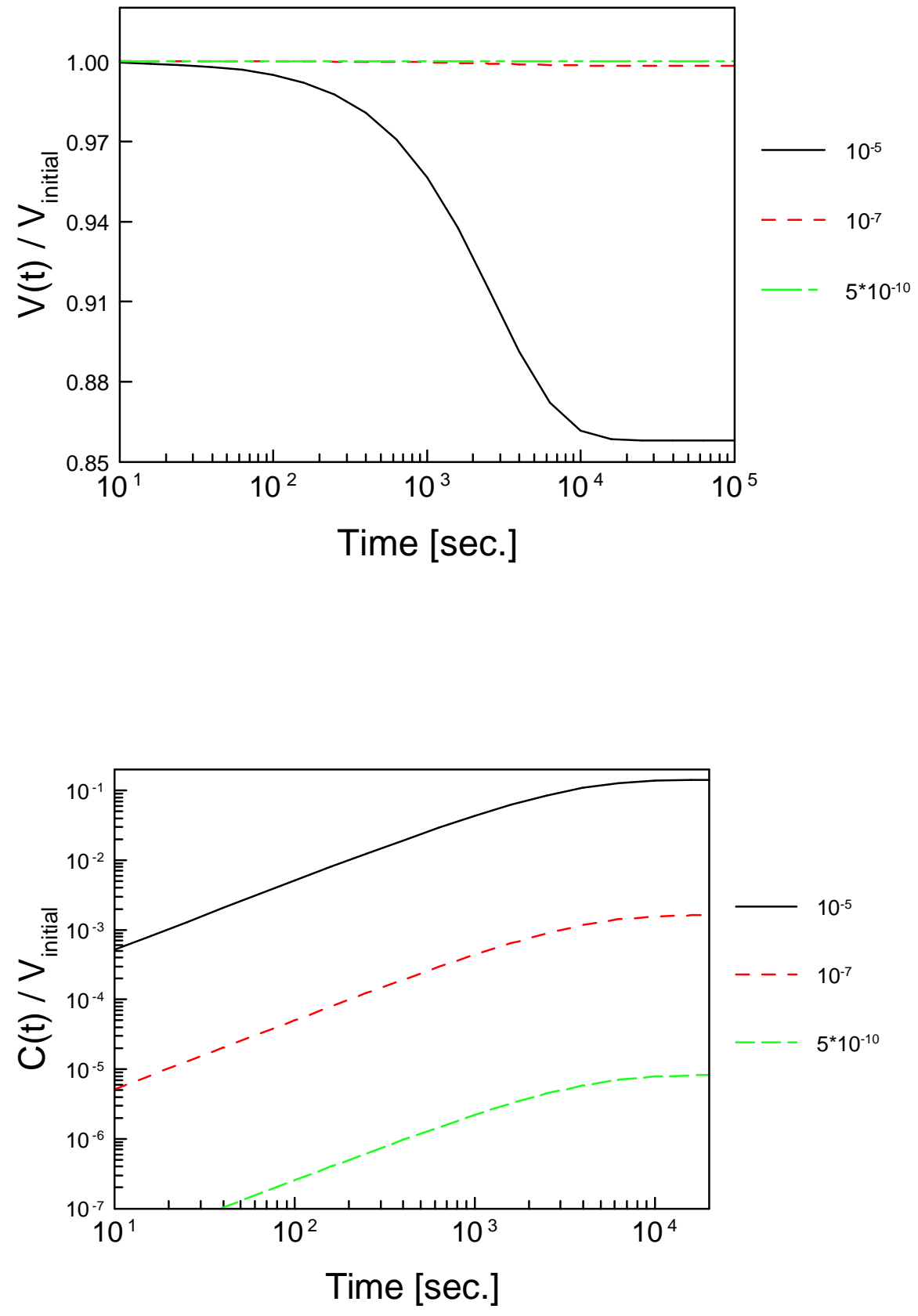